\title{
Hermann Danuser, Metamusik, Schliengen: Edition Argus 2017
}

Schlagworte/Keywords: meta-music; Metamusik; music aesthetics; music theory; Musikästhetik; Musiktheorie; referentiality; Selbstreferentialität; self-referentiality

Metamusik: Nach Mitteilung des Verlags ist das »alles mit Musik Verbundene, das jenseits des Hörbaren existiert [...]. « ${ }^{1}$ Über Musik im engeren Sinne, ein bloß erklingendes und womöglich noch notiertes Kunstgebilde, geht Metamusik freilich hinaus. Als erklingende und notierte wäre Musik in Metamusik einbezogen, wo sie mindestens eine zweite Dimension beinhaltet, und sei es eine aus derselben Existenzform, zum Beispiel Klang. Was sie zu Metamusik macht, ist bei Musik über Musik die reflektierende Verdoppelung, als Denk- oder Vorstellungsmoment jenseits des Hörbaren. Simpler in Analogie zu Metaphysik als jenseits der Physis liegend (oder schwebend), wäre Metamusik auch Alltägliches wie Reden oder Schreiben über Musik, Malen zu oder nach Musik. ıMetas wären Tätigkeiten wie Musik »erfinden, erforschen, notieren, beschreiben, malen, sehen, denken und sich vorstellen « (24), manchmal bei verdoppelter erster Existenzform - sogar hören, singen, spielen. Solche musikalischen Selbstreflektionen, Selbst- oder Metareferenzen der Musik liefern den Stoff und Titel für Hermann Danusers Metamusik. Dass es sich um ein Projekt handelt, welches ihn »seit der [...] Studienzeit« (7) beschäftigte, mag einer der Gründe dafür sein, dass seine Metamusik aus der Zeit fällt. Aktuelle Diskussion zu Metareferenzen in Musik, initiiert vor allem von literaturwissenschaftlicher Seite, lässt Danusers Buch nicht beiseite, hakt sie aber geschwinde ab. Und solche Nebenschauplätze von Musiktheorie lasse auch ich im Folgenden aus dem Auge und lese das Buch wie einen Roman, der für etliche Passagen mein Fachgebiet behaust.

Schon als Gegenstand ist das Buch ganz unmetaphysisch eine Kostbarkeit: Das Format knapp unter dem A4 von Arbeitsheften - und damit eben doch ein großes Buch. Dunkellilabrauner Umschlag, aus einer bestimmten Per-

1 http://www.editionargus.de/assets/own/796.htm\#Zusammenfassung (26.4.2020). spektive und bei passendem Licht wird eine Maserung sichtbar. Auf dem Buchrücken kupferfarben eingeprägt Autorenname und der umfassend-kurze Titel, der Frontdeckel nur mit dem Verlagssignet: breit lidschattig eines von hundert Argusaugen. Dunkelgelbgoldenes Lesebändchen, das hellpappige Vorsatzpapier will berührt sein. Auf dem letzten Blatt vorm hinteren Vorsatzpapier, also auf Seite 499, informiert der Verlag über die Materialien und über die Hersteller der Einzelbestandteile der Metamusik - als physischem Gegenstand. Von den beinahe 500 Seiten dieses Opus magnum enthalten die letzten 25 ein Namen- und Werkregister (Namen in Fußnoten sind nur dort einbezogen, wo es um mehr als ums Bibliographieren geht), Nachweise der auch farbigen Abbildungen und eine Übersicht der Vorarbeiten, aus denen der Text entstand. Für den Aufbau des Buchs - oder für das Musikweltgebäude, das es errichtet, - stand Jean Paul Pate. Zweieinhalb Seiten Vorwort, datiert mit Oktober 2016, 40 Seiten Einleitung (11-50), dann die Hauptteile I bis IV: 60 Seiten Teil I über »Metareferenzen « (54-113), vorwärts durch die Geschichte 137 Seiten Teil II über »Metamusiktheater « (116-252), rückwärts durch die Geschichte 163 Seiten Teil III zu »Verkehrte[r] Geschichte« (256-418) und 52 Seiten Teil IV über »Selbstreflexives Hören, Sehen, Denken, Schreiben« (422-474). Systematisches wird mit Historischem verstrickt, damit die Textur des Buches selber Erkenntnis verschaffe. Es gibt eine lange Introduktion, dann die vier Teile. Die äußeren (systematischen) Teile entsprechen einander von der Länge her ungefähr, auch haben sie je gleich viele Kapitel (fünf), die inneren (einmal vorwärts, einmal rückwärts diachronen) Teile haben wieder gleich viele Kapitel (zwölf, aufgeteilt in je viermal drei) und in etwa gleiche Länge. Dem Episodischen der Mittelteile gewährt Danuser ungleich viel Raum (wie bei einer Mahler-Symphonie). In sich sind die Teile gebaut wie jene Bilder, von 
denen der Autor besonders gerne spricht: solche mit Bildern und Spiegeln in Bildern.

Je ein metamusikalisches Gemälde von Juan Gris eröffnet als Frontispiz die Einleitung und die vier Teile: Violine und Schachbrett (10), für die "Metareferenzen" Le Cahier de musique, postiert auf einem Blatt unbeschriebenen Notenpapiers, eine Gitarre im Hintergrund (52), für »Metamusiktheater « ein Harlekin mit Gitarre, von der zwei Wirbel zu erkennen sind (114), für »Verkehrte Geschichte" Die Gitarre, an die Wand gehängt neben ein anachronistisches Bild im Bild - Henry Howards The Swiss Peasant - (254), ${ }^{2}$ und Der Tisch des Musikers, vor nochmals einer Gitarre ein diesmal beschriebenes Blatt Notenpapier, halb verdeckt eine Lyra (420). Die Bilder sind dezent mattfarbig und vertrackt-klassisch. Metamusik ist insgesamt reich bebildert, auch gibt es zahllose Notenbeispiele, mal als Reproduktionen von Autographen wie von Arnold Schönbergs unvollständig gebliebener Klavierfassung des Orchesterliedes Natur aus seinem Opus 8 (107), mal neu gesetzt wie der Ausschnitt aus Ranieri de' Calzabigis (als Librettist) und (als Komponist) Florian Leopold Gassmanns L'opera seria von 1769. Der Ausschnitt weist ein seltener Fall - einen (wohl schon aus der Vorlage übernommenen) Eintrag in den Noten auf (132, T. 70), ansonsten sind die Notenbeispiele - eine ästhetischer Entscheidung - pur angefertigt, ohne analytische Einträge. Gassmanns metamusikalische Maßnahmen gehören zu den wenigen von Danuser ausführlicher besprochenen Produktionen, die nicht von einer Größe westlicher Tonkunst ${ }^{3}$ stammen, die Größe ist hier der Koproduzent Calzabigi. Die Maßnahmen seines Librettos zu dieser Oper über die Oper vereinen (mindestens) vier der metakünstlerischen Kontext-Modi, die Danuser auseinanderhält: einen extratextuellen, einen produktionsästhetischen, einen intratextuellen und einen rezeptionsästhetischen (127). Die

2 Ich danke Daniel Serrano für die Ermittlung des Namens des Malers und des Bildtitels.

3 Auf diese Eingrenzung weist auch Frieder von Ammon hin. In Metamusik entstehe ein »Panorama der vor allem europäischen, um die kanonischen Komponisten und Werke zentrierten Musikgeschichte«. (Ammon 2019, 525, linke Spalte) durch und durch zeitgenössische Musik Gassmanns erhält erst durch die literarischen Maßnahmen ein Darüberhinaus, sie rückt in die Frühgeschichte der Moderne, passend zu Juan Gris' Gemälden (10). Tatsächlich bietet Danuser, wie an dem Bespiel ersichtlich, ein komplexes Theorie-Gestänge aus vier plus sieben an. Es gebe zwei Prämissen dafür, dass sich von Metamusik reden lasse. Eine ist, dass man es mit auf Musik bezogenen Textkategorien zu tun haben müsse. Danuser unterscheidet dafür vier gleichberechtigte Existenzformen: Klang, Notation, Wortschrift und Bild (25). Die andere Prämisse ist, dass sich diese Textwerdung über Klang, Notation, Wortschrift oder Bild im Werk und seiner Performanz realisiere (38). Dafür unterscheidet Danuser sieben Modi von Kontext-Begriffen: den intratextuellen, infratextuellen, intertextuellen, intermedialen, extratextuellen, produktionsästhetischen und den rezeptionsästhetischen (25 f. sowie, en détail erläutert, 31-38). Diese Definition erlaube nicht, Musik und Metamusik "glasklar " voneinander zu trennen (25), oft lässt sich Musik selber als Metamusik begreifen, aber es werden doch Geltungskreise umrissen. Danuser ergänzt seine Ausfaltung des 4+7-Gestänges schließlich »aus persönlicher Sicht«: Erinnerungen an Metamusik-Festivals und Installationen in der Galerie Gelbe Musik im Berlin der mittleren 1970er Jahre (38). Wesentlich war für diese die Durchlässigkeit von Klang, Bild und Buchstabe. $\mathrm{Zu}$ Performances der letzten Jahre, bei denen Technik mehr Terrain okkupiert, stößt Danuser die Tür weit auf (91, Anm. 92, und 95-98).

In extenso (auf gut acht Seiten, 42-50) erläutert er die Gliederung oder den Plan seiner Metamusik, den er mit einer Parallelbildung zum Alten Testament in charmanter Selbstüberhebung und Mut zum Scheitern »Buchbau « nennt. Der Turmbau zu Babel beschließt eine Serie menschlicher Verfehlungsgeschichten, ihm folgt die Verwirrung der Sprachen auf dem Fuße, was soll man da noch mit der einen Zunge des Volks aus dem Osten reden? Danuser will keine Fachterminologie durchsetzen, er liebt frei flutende Prosa. Die Darstellung des Diskussionsstandes über Metamusik beendet er mit kritischen Bemerkungen zu Werner Wolfs medientheoretischem Konzept, das ihm "zumindest stark verkürzt" vorkommt (38, Anm. 52, 43). Er selber verfolgt »weniger semio- 
tische als kunstwissenschaftliche, mehr musikals medientheoretische Ziele«. (42) Und so trifft man auf die ehrwürdige Tonkunst. Metamusikalisch kontextualisiert kann Tonkunst »innerund außerhalb von Werken « werden (ebd.). 'Tonkunstı und 'Werke ‘ öffnen sich aber gen Satire, Machwerke, Prozesse von künstlerischer Forschung (91 f.) oder die Metamusik von Pädagogik (98-101).

Danuser praktiziert einen erweiterten, oft sogar aufgelösten Musikbegriff. ${ }^{4}$ Lange Jahre war er im Kuratorium der Ernst von Siemens Musikstiftung; seine Mitgliedschaft im Stiftungsrat und wissenschaftlichen Team der Paul Sacher Stiftung - seit undenklicher Zeit, möchte man sagen - haben damit zu tun (und dafür gesorgt), dass er weiß, wie der Hase läuft. Das Thema Referentialität fordert (den klugen Igel) dazu heraus, dass er, der Metamusiker, den sechsten und siebten metakünstlerischen Kontext-Modus, den produktions- und den rezeptionsästhetischen, am eigenen Spiegelbild erprobt. Das kommt einem gefährlich vor. Danusers Buch heißt, was es ist. Nicht nur, weil über Musik geredet wird, sondern weil der Text zu einem Kunstgebilde wurde, zu einem MetaTonkunst-Stück. Das bleibt freilich durchlässig und kapselt sich nicht ab. Im letzten Kapitel, »Metamusikers Spiegelbild« (462-474), resümiert Danuser seinen Werdegang als Musiker und als Wissenschaftler (und ein wenig auch den familiären). Er macht das Kapitel sehenden Auges zu einem "Grenzfall von Wissenschaft" (50), wie man an die Grenze des Anstands gerät, wenn man von sich statt von der Sache redet. Sich von Tonkünstler*innen anzuhören, dass ihr Leben sich in Kunst verwandelte oder wie ihre Kunst ihr Innerstes hervorkehre, kann aber Interpret*innen zu Improvisator*innen überlaufen lassen. Dort waschen sie nicht mehr anderer Leute Seelen aus. ${ }^{5}$ Bei einem Wissenschaftler nähere der öffentlich präsentierte Blick in den Spiegel sich »einer Entgleisung " (50) und ist kein Normalfall. Wunderbar, dass

4 In die Diskussion um die »Erweiterung des Musikbegriffs, die Hinwendung zu Musiktheater und Performance, de[n] Streit um den konzeptuellen Gehalt von Musik« greife Danusers Buch ein, ohne sie explizit zu verhandeln (Haffter 2018, 49, mittlere Spalte).
Danuser den Anstand für ein Weilchen vergaß. Indem er den Blick auf den Verlauf seiner Karriere und sogar auf Privates freigibt, kreiert der Autor für sein Opus magnum, analog zum Thema des Buchs, einen erweiterten Wissenschaftsbegriff. Mit den Erweiterungen von Wissenschafts- und von Musikbegriff nimmt die eine - immer sozusagen beiseite geredet Züge der anderen an. Tonkunst bringt Verweise und schämt sich nicht dafür, Wissenschaft tut nicht so, als sei sie vom Himmel gefallen. Zu dem im Schlusskapitel hervorgekehrten produktionsästhetischen Modus gelangt, durchgehend in die Metamusik eingewoben, der persönlich-rezeptionsästhetische; Danuser schmuggelt inn nicht anonymisiert durch. Warum gibt er an, dass er bestimmten Aufführungen von Mahlers Neunter Symphonie Mitte der 1990er Jahre in Amsterdam und Berlin »beiwohnte« (63)? Die Berliner Philharmoniker, geleitet von Claudio Abbado, dehnten eine Passage aus dem Finale dort »ins kaum noch Hör- und Darstellbare " und ließen »den Klang ins nicht mehr Realisierbare gleiten «. (ebd.) Protokolliert ist, wer wen wann mit welchem Stück und wo hörte. Dass Dieter Schnebel den Zeithorizont von Mahlers Sinfonik im Mahler-Moment (1986) aus seinem Zyklus Re-Visionen II ins Unendliche dehnte, lässt entlegene Zeiten und lässt Produktion mit Interpretation und Rezeption zusammenfallen. 1910 (das Jahr der Fertigstellung der Neunten) und die Jahre um 1989 (Schnebels Moment, Abbados Dirigate und Danusers Konzertbesuche) stecken Anfang und Ende der Neuen Musik (und zugleich des kurzen 20. Jahrhunderts) ab. Nicht expliziert ist, dass der Komponist (Schnebel), der Dirigent (Abbado) und der Hörer (Danuser) in jenen Jahren in derselben Stadt lebten. Derselbe Ort zur fast selben Zeit (Ende der Mauerstadt Berlin) tritt in Parallele zu Mahlers Toblacher Kompositionszeit (im Endstadium des Imperialismus). Danuser bezieht mündliche Äußerungen Schnebels ihm gegenüber ein (61, Anm. 23), aber er traut Komponistenmitteilungen natürlich nicht. Wann dürfen Leser*innen der Metamusik Äußerungen des Wissenschaftlers Danusers trauen? Und sie nicht nur als persönliches Erleben abhaken? Wegen der exakten Protokollierung und der persönlichen Markierung möchte ich glauben, dass eine Aufführung des von Abbado geleiteten Lucerne 
Festival Orchestra von Mahlers Erster Symphonie am 20. September 2009 in Beijing (hier fehlt der Konzertsaal), wo Danuser mit seiner Frau zugegen war (440, Anm. 62), obwohl (oder gerade weil) nicht richtig geprobt worden war, den »Rang des Außerordentlichen « (439) erreichte und dass die Aufführung von Mahlers Sechster Symphonie am 4. Juli 2004 in der Berliner Philharmonie mit den Berliner Philharmonikern, wieder geleitet von Abbado, den Sinn von Theodor W. Adornos Formulierung aufgehen lassen konnte, Mahlers Musik sei »konkret zur Idee bestimmt" (440, Anm. 62).

"Serien, Reihen, Gruppen " (456) ermöglichen das Musikherstellen erst und hemmen es nicht. Darauf hätte man sich einzulassen, und für die musikologische Produktion gelte das »übrigens auch" (457). Aus »meiner Sicht» muss "ein Musikwissenschaftler, um zum Kern zu gelangen, sich in die zu deutenden Werke hineinversetzen « (ebd.). Hineinversetzen mag Hineindenken und das alte Einfühlen umschließen. Verfahren, bei denen man draußen sitzen bleibt, sich nicht einlässt, kommen im buchstäblichen Sinne nicht in Betracht. Orientiert Musikologie sich aber am "Klangakt» (462), d. h. an dem, wohin musikalische Kunstwerke von sich aus und was sie von sich aus werden wollen (eine von Horst Bredekamps Theorie des Bildakts angeregte Neuakzentuierung von ‘Kunstwollen`, wenn man möchte), hat sie »die Chance, Ergebnisse von einiger Relevanz zutage zu fördern« (ebd.). Zu denken geben darf das Musiktheoretiker*innen bei der Konzeption von Formenlehren, bei Korpusstudien, beim Nachvollzug des Fortwirkens der Generalbassharmonik bis ins 19. und 20. Jahrhundert hinein. Um nicht missverstanden zu werden: Von den Subjekten von Komponist*innen bleibt das Gewusel im Kunstwerk selber im besseren Falle unberührt.

$\mathrm{Zu}$ solchen »Kunstreflexionen « (Teil der Überschrift von Teil IV, Kapitel 4) geben dem Verfasser von Metamusik Adornos Schreiben über und Reden von Musik und deren Verhältnis zur deutschsprachigen universitären $\mathrm{Mu}$ sikwissenschaft seiner Zeit Anlass. Adorno ließ seine Texte schön klingen, ihr Mix von Gediegenheit, Melancholie und Frechheit sollte überraschen. Nur hätte die Einsicht, dass jeder Künstler oder Denker im gesamtgeschichtlichen Strom schwimmt (wenn er nicht unterge- hen will), keine Selbstüberhebung aufkommen lassen dürfen. Das übersah Adorno für seine eigene Person. Ihm war »Eitelkeit nicht fremd « (458), wie Danuser es dezent formuliert - mit einer entsprechenden Bemerkung hatte Rudolf Stephan, der den Philosophen noch dazu aus dem persönlichen Gespräch kannte, seine Hörer*innen erlöst, wenn sie sich aufrieben an dem Verständnis gewisser Passagen, die sich wunderbar anhörten, aber merkwürdig unsachgemäß und unnachprüfbar waren. Danuser aber mutet sich manches zu, manchmal auch ohne Rücksicht auf sein Spiegelbild. In den 1970er Jahren traute er sich, den Klavierpart von Charles Ives' Violinsonaten aus Kopien der Manuskripte in der ersten angesetzten Probe vom Blatt zu spielen. Auch als Musiker und Musikologe ist er »kein Kostverächter « ${ }^{6}$ In der Metamusik nährt er sich immer wieder von Kunst, deren Ruhm begrenzt ist. Zu Normalkostmusiken, mit denen er sich befasst, gehören die von Gassmann oder die frühen vierhändigen Militärmärsche von Franz Schubert. Zu unverdaulicher musikalischer Kost gehören etwa die Rätsel in Pietro Cerones Melopeo (1613). "Bescheidenheit war [Cerones] Sache« trotzdem nicht (393). Abgesehen von dem kommerziellen Effekt, den Anpreisen haben konnte und das Verlagshäuser daher einforderten, so dass es wohl nicht auf Cerones Konto ging, braucht er sich im Himmel nicht nach-

6 So drückte Rainer Sachtleben es aus. Er hatte sich mit Danuser durch Ives' handgeschriebene Noten gekaut. Als Vorkoster half er in den 1980ern bei Aufführungen von Hanspeter Kyburz, Isabel Mundry und anderen Gösta Neuwirth-Schülern aus, wenn »die Stücke, die wir geschrieben hatten «, von den »Studenten nicht gespielt werden konnten«. (Kyburz in Grünzweig 2019, 107) Danuser verfolgt »Verkehrte Geschichte» zurück bis zu Josquin des Prez. Hier zeigt er sich gegenüber metamusikalischen Dimensionen in Gestalt gematrischer Summen skeptisch. Deren geschichtlich verkehrtes Potenzial liegt zutage und hat über Neuwirths (von Danuser nicht erwähnte) Deutungen, mehr vermutlich über die mündliche Lehre als über Geschriebenes, Kompositionsgeschichte gemacht. Noch vor Kurzem hielt der Neuwirth-Schüler Peter Ablinger auf seiner Homepage einen besonders schlechten, wohl uralten Scan von Neuwirths »Erzählung von Zahlen« (Neuwirth 1982) herunterladbar vor. 
träglich zu schämen, denn Danuser feiert die Rätselnotenbilder seines Traktats der theoretischen und praktischen Musik als "eine wahrhaft avantgardistische Alte Musik«, verwandt mit graphisch notierter Musik der 1950er bis 1960er Jahre (401). Bei Cerones Rätseln sei "geradezu irrelevant«, wie sie gelöst klängen. Sie gingen des Klangs verlustig, wiesen aber immerhin drei von vier Existenzformen von Metamusik auf: „Notation, Erläuterung, Bildgestalt«. (ebd.) Die Parallele zur Konzeptkunst der letzten Jahre zieht Danuser nicht, legt sie aber nahe, wenn er den zeitgenössischen spanischen Conceptismo erwähnt. Stattdessen spielt er mit »Old Complexity« (ebd.) für Cerones Bilderrätsel auf Brian Ferneyhough und dessen Deutung durch Claus-Steffen Mahnkopf an, ohne einen der Namen fallen zu lassen. Das darf als Dezenz gedeutet werden.

Auch auf lebende Musiktheoretiker*innen nimmt der Autor, soweit ich sehe, kaum Bezug. Angeführt werden Ariane Jeßulats Dissertation über Die Frage als musikalischer Topos und ihre Habilitationsschrift Erinnerte Musik (181), auch Ludwig Holtmeiers Bemerkungen zum Fortleben von Hugo Riemanns Funktionsbezeichnung in der bundesdeutschen musiktheoretischen Alltagspraxis (435, Anm. 47) und die »Neo-Riemannians", die seit Jahrzehnten zu jener Musik vordrängen, die »ihr Patron $[\ldots]$ in die Verbannung geschickt hatte« (435), namentlich Richard Cohn mit seiner Topographie der Harmonik in den Jahren von Sigmund Freuds Traumdeutung (oder weniger assoziationsreich: in Wien um 1900). Wenn Danuser sich auf musiktheoretische Diskurse der letzten Jahrzehnte kaum bezieht, können umgekehrt Musiktheoretiker*innen (abgesehen von Allgemeinbildungsinteressen) in der Metamusik gleichwohl Anknüpfungspunkte für ihr Fach finden. Besonders dem Kapitel über Pierre Boulez dürfte ihr Interesse zufliegen, unter anderem wegen der Reproduktion von Manuskripten aus dem Bestand der Paul Sacher Stiftung (z. B. die Abbildungen 9a und b, 10 und 12 auf den Seiten 278 bis 280 und 285). Die Spannung von Rationalität und Phantasie, die Danuser als hervorstechend für Boulez ausmacht, fordert die Reflexion über den eigenen analytischen Zugang heraus und lädt zu Detailstudien ein.
Dass Boulez chronologisch zurückschreitend (und damit die Idee verkehrter Geschichte in die Anordnung umsetzend) zusammen mit Wolfgang Rihm vor ihm und Anton Webern nach ihm in das Großkapitel "Konservative Neue Musik « aufgenommen wurde (und dessen Herzstück bildet), verdankt sich der Tatsache, dass Boulez das Zurückschreiten lebenslang im eigenen Werk vollzog, beispielsweise indem die Notations für großes Orchester, eine teilabgeschlossene Produktion des alten Künstlers, eine des ganz jungen reflektieren. ${ }^{7}$

Eine formale Nebenbemerkung: Die Zahl von Tippfehlern oder Versehen in Danusers Opus magnum hält sich in sehr engen Grenzen: mal ein Buchstabe zu viel ("scheiterete", 434), mal ein offensichtlicher Unfall (260), ${ }^{8}$ mal ist eine Datumsangabe irritierend: »nach Dahlhaus' Tod im Winter 1989«(468); er starb am 13. März, als der kalendarische Winter zu Ende ging. Für zentrale Begriffe wäre eine einheitliche Schreibweise wünschenswert gewesen: Mal schreibt Danuser Kontext-Begriff (z. B. 25), mal Kontextbegriff (38), mal Text-Kategorie (z. B. 24), dann wieder Textkategorie (38).

Metamusik greift auf 35 Texte zurück, die in der Mehrzahl aus Vorträgen hervorgingen und die zu einem größeren Teil bereits veröffentlicht wurden, der früheste darunter 1981, die beiden jüngsten 2015. Trotzdem ist Metamusik nicht bloß eine Zusammenstellung anderswo (vielleicht entlegen) publizierter Texte mit einigen nicht publizierten Vortragsmanuskripten. Denn nicht nur seine früheren Texte hat Danuser grundlegend umgearbeitet (oder ausgefaltet, um den Begriff für Boulez' Verfahren aufzunehmen). In anderer Version erst 2010 publiziert wurden beispielsweise die Gedanken über »Riemanns Tontheorie» (422-435). Die auf einen Vortrag beim Jahreskongress der Gesellschaft für Musikforschung in Leipzig 2008 zurückgehende Fassung des Metamusik-Kapitels erschien in der Zeitschrift der Gesellschaft für Musiktheorie. ${ }^{9}$ Praktisch kein Satz blieb aber gleich, es gibt Zuspitzungen und damit Verknappungen, dann wieder willkommene Erweiterungen, wie zu dem von Riemann umnotier-

\footnotetext{
7 Siehe dazu auch Grabow 2016

8 Auf diesen wies bereits Ammon (2019, 525, rechte Spalte) hin.

9 Danuser 2010
} 
ten Lied Max Regers. Wie die neu hinzugefügten Notenbeispiele zeigen (433), sortiert Riemann die im oberen System geschriebenen Töne in Stimmenkomplexe um und ergänzt Phrasierungen, so dass parallele Oktaven zwischen Tönen der rechten und denen der linken Hand nach Registrierungen und damit erlaubt aussehen, die Harmonik ordnet er durch enharmonische Verwechslungen in seinem Sinne, dynamische Angaben sind hinzugefügt, so dass der - nach Cohns Terminologie mit den hexatonischen Polen das Unheimliche signifizierende - Umschlag von B-Dur (bzw. in Riemanns Schreibweise Ais-Dur) nach fis-Moll dynamisch extrapoliert wird und nun als das aufgeschrieben ist, wohin Riemann über den Gegenkleinterzwechsel gelangt. ${ }^{10}$

Riemann durfte in Danusers letztem Metamusik-Teil, der Hören, Sehen, Denken und Schreiben als Modi von Selbstreflektion behandelt, das Kapitel zum Hören vertreten. Das Kapitel über Sehen vertritt Herbert von Karajan. Man ahnt, dass er nicht gut wegkommen wird bei jemandem, der von Abbado schwärmt. Nur wie gelingt es ihm, das Unbehagen an dem »blinden Demiurgen« (439) verständlich, nachvollziehbar, ja sogar fühlbar zu machen? Das Kapitel ist ein Meisterstück des Redens über musikalische Interpretation. Riskant mag es sein, eigens Erfahrenes und Gehörtes einzubringen (»Eine Erinnerung aus meiner Jugendzeit [...]. [...] sagte mir in privatem Kreis ein Philharmoniker«, 439). Widersprüche zusammenbindende Formulierungen kommen aber zupass: Karajan war ein »performative[s] Genie, das die Performance-Kunst bekämpfte« (445). Dass »sich nach Karajans Tod Ruhe» ausbreitete, deutet Danuser mit der Vergänglichkeit von dessen Idealen. Uns zum Vergnügen spielt der Autor in der angehängten Passage (»trotzdem bleibt noch viel zu entdecken«, ebd.) noch einmal die Sphinx. Zu seiner Praxis gehöre eine gewisse Passivität, er reagiere lieber, als dass er agiere (473). Die Fragen sind gestellt. Das Antworten liegt bei uns.

\section{Gesine Schröder}

\section{Literatur}

Ammon, Frieder von (2019), »Hermann Danuser, Metamusik« [Rezension], Die Tonkunst 13/4, 525-527.

Danuser, Hermann (2010), „Von unten und von oben? Hugo Riemanns reflexive Theorie in der Moderne«, Zeitschrift der Gesellschaft für Musiktheorie 7, Sonderausgabe, 99-116. https://doi.org/10.31751/564 (26.4.2020)

Grabow, Martin (2016), Erfindung, Recycling, Neukomposition. Untersuchungen zur inneren Verflochtenheit des Lebenswerks von Pierre Boulez am Beispiel der "notations", Hildesheim: Olms.

Grünzweig, Werner (2019), »Hanspeter Kyburz«, in: Wie entsteht dabei Musik. Gespräche mit sechs Komponisten und einer Komponistin über ihre Studienzeit, Neumünster: von Bockel, 79-107.
Haffter, Christoph (2018), „Hermann Danuser, Metamusik« [Rezension], Dissonance 142, $49 \mathrm{f}$.

Neuwirth, Gösta (1982), „Erzählung von Zahlen«, in: Josquin des Prés (= MusikKonzepte, Bd. 26/27), hg. von Heinz-Klaus Metzger und Rainer Riehn, München: edition text + kritik, 4-38.

Riemann, Hugo (1920), Handbuch der Harmonie- und Modulationslehre (Praktische Anleitung zum mehrstimmigen Tonsatz), 8. Auflage, Berlin: Hesse.

Yu, Ying (2020), "Auf Schatzsuche». Improvisatorische Aufbereitung zeitgenössischer Klaviermusik, Masterarbeit, Hochschule für Musik und Theater »Felix Mendelssohn Bartholdy« Leipzig.

10 Vgl. z. B. Riemann 1920, 112, Nr. 12 der Liste: $\mathrm{C}^{+}-{ }^{\circ}$ es oder in Funktionszeichen $\mathrm{T}-\left({ }^{\circ} \mathrm{S}\right)^{\circ} \mathrm{Tp}$. 
Schröder, Gesine (2020): Hermann Danuser, Metamusik, Schliengen:

Edition Argus 2017. ZGMTH 17/1, 177-183.

https://doi.org/10.31751/1040

(C) 2020 Gesine Schröder (gcm.schroeder@gmx.de)

Hochschule für Musik und Theater >Felix Mendelssohn Bartholdy` Leipzig [University of Music and Theatre ,Felix Mendelssohn Bartholdy< Leipzig ]

Dieser Text erscheint im Open Access und ist lizenziert unter einer Creative Commons Namensnennung 4.0 International Lizenz.

This is an open access article licensed under a

(c) (1)

Creative Commons Attribution 4.0 International License.

eingereicht / submitted: 16/03/2020

angenommen / accepted: 16/03/2020

veröffentlicht / first published: 15/06/2020

zuletzt geändert / last updated: 15/06/2020 